\title{
Phosphorylated MLKL and cleaved caspase8 are useful for early diagnosis of Stevens-Johnson syndrome and toxic epidermal necrolysis.
}

\author{
Akito Hasegawa, Manami Maehara, Nanase Yamazaki, Yohya Shigehara, Riichiro Abe \\ Division of Dermatology, Niigata University Graduate School of Medical and Dental Sciences, Niigata, Japan.
}

\section{Introduction}

Stevens-Johnson syndrome (SJS) and toxic epidermal necrolysis (TEN) are rare but extremely severe and life-threatening cutaneous adverse drug reactions. It is difficult to distinguish erythema multiforme (EM) and SJS/TEN in early stage.

Histopathology shows keratinocyte death in SJS/TEN and EM in various frequency. Although keratinocytes have been suggested to die as apoptosis, our recent study revealed necroptosis, programmed necrosis, also contribute to keratinocyte death in SJS/TEN. Monocytes secrete Annexin A1 in stimulation of causative drugs. The released annexin A1 binds to FPR1 and mediates the RIP1/RIP3 complex. MLKL is phosphorylated by RIP3 and initiate necroptosis ${ }^{1}$. Since necroprotosis is considered to be specific to SJS/TEN, we hypothesized that analyzing the form of keratinocyte death is useful for early diagnosis of SJS/TEN.

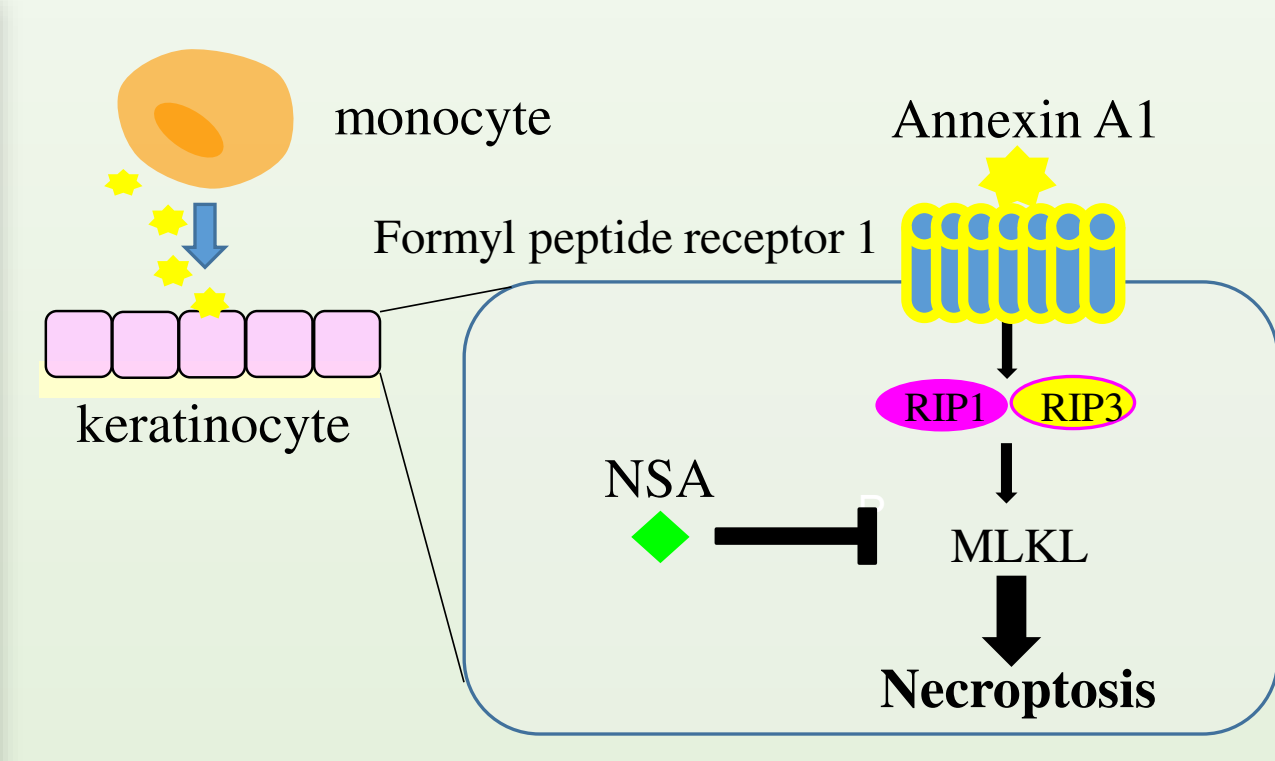

Saito N et al. Sci Transl Med 2014

\section{Materials and Methods}

The skin samples were obtained from non-bullous erythematus lesion of the patient with acute phase drug eruption.

We included 4 patients diagnosed as SJS and 10 patients diagnosed as EM. We performed immunofluorescence staining. Anti-phosphorylated MLKL (pMLKL) staining was used to detect necroptosis, and anti-cleaved caspase8 (cCas8) staining was used to detect apoptosis.

The number of death keratinocyte in H-E staining and pMLKL and cCas8 positive cell in immunofluorescence staining were counted.

\section{Result}

No Age Sex

cause skin rush area $(*)$

Musosal involvement Other symptoms Diagnosis

S1 $28 \quad \mathrm{M} \quad$ acetaminophen Conjunctival injection

S2 $23 \quad$ F $\quad$ drug for common cold $\quad 4+$ Erosion of lip and oral mucosa liver injury SJS Congestion, corneal erosion Erosion of oral mucosa liver injury SJS

S3 $\quad 65 \quad \mathrm{~F} \quad$ tegretol

S4 $67 \quad$ F $\quad$ loxoprofen $\pm \quad$ Erosion of lip and oral mucosa

$-$
SJS

\section{E1 $75 \mathrm{M} \quad \begin{gathered}\text { acetaminophen or } \\ \text { tranexamic acid }\end{gathered}$} $3+$ Conjunctival injection Erosion of lip and oral mucosa liver injury E2 $67 \quad \mathrm{~F} \quad$ salazosulfapyridine $3+$ Conjunctival injection Erosion of oral mucosa EM $4+$

*skin rush area 0-20\%: $\pm, 20-40 \%: 1+, 40-60 \%: 2+, 60-80 \%: 3+80-100 \%: 4+$

In case of EM, 2 of 10 cases were shown. One was only pMLKL positive and the other was both pMLKL and cCas8 were negative.

No S1
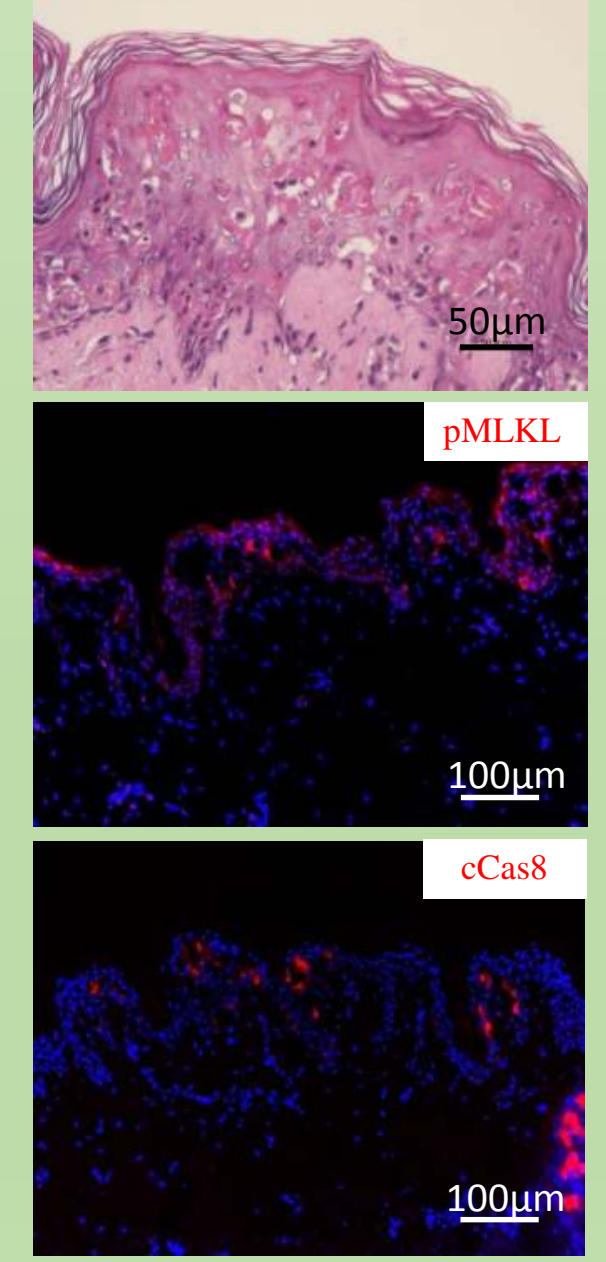

No S2
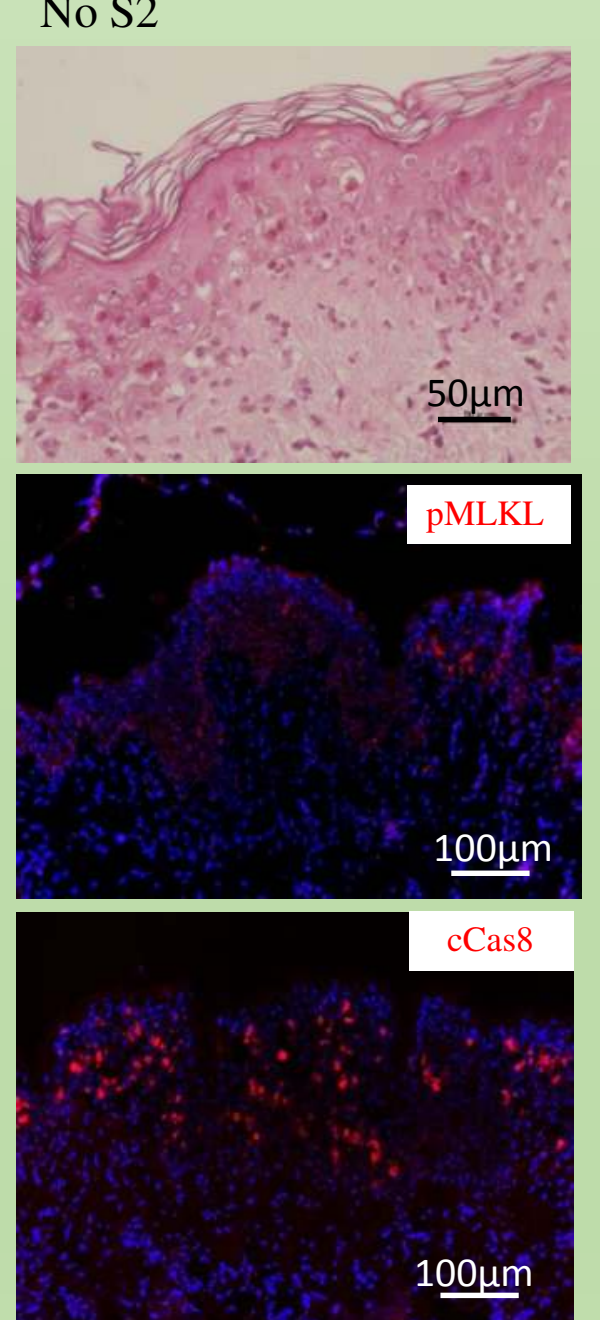

No S3
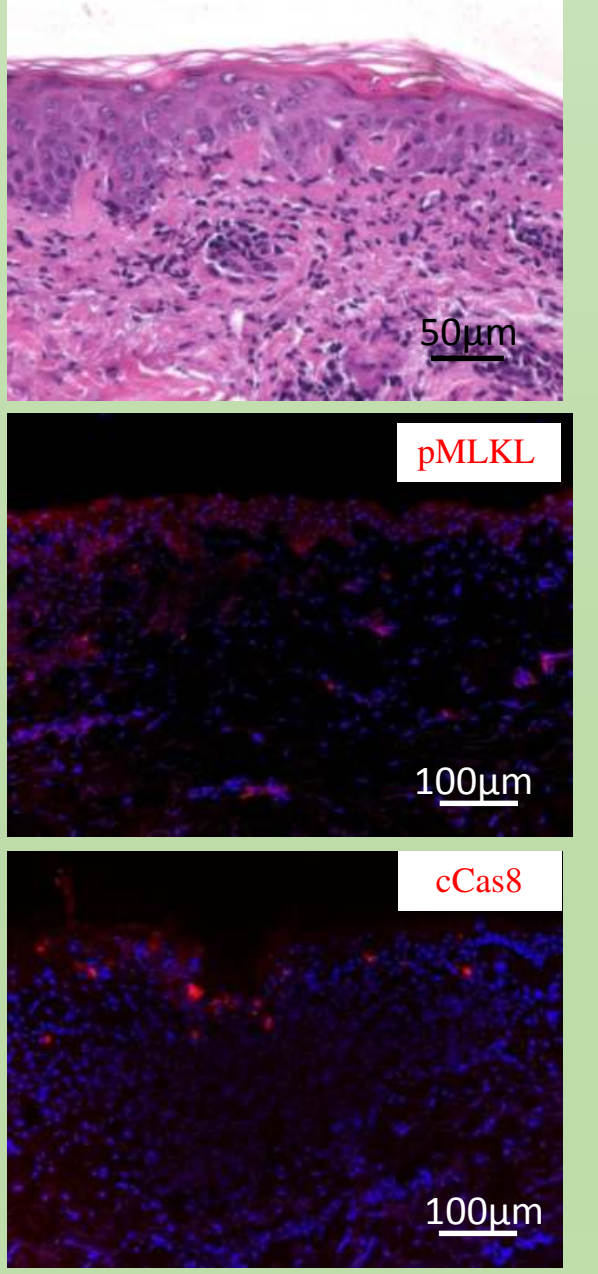

No S4
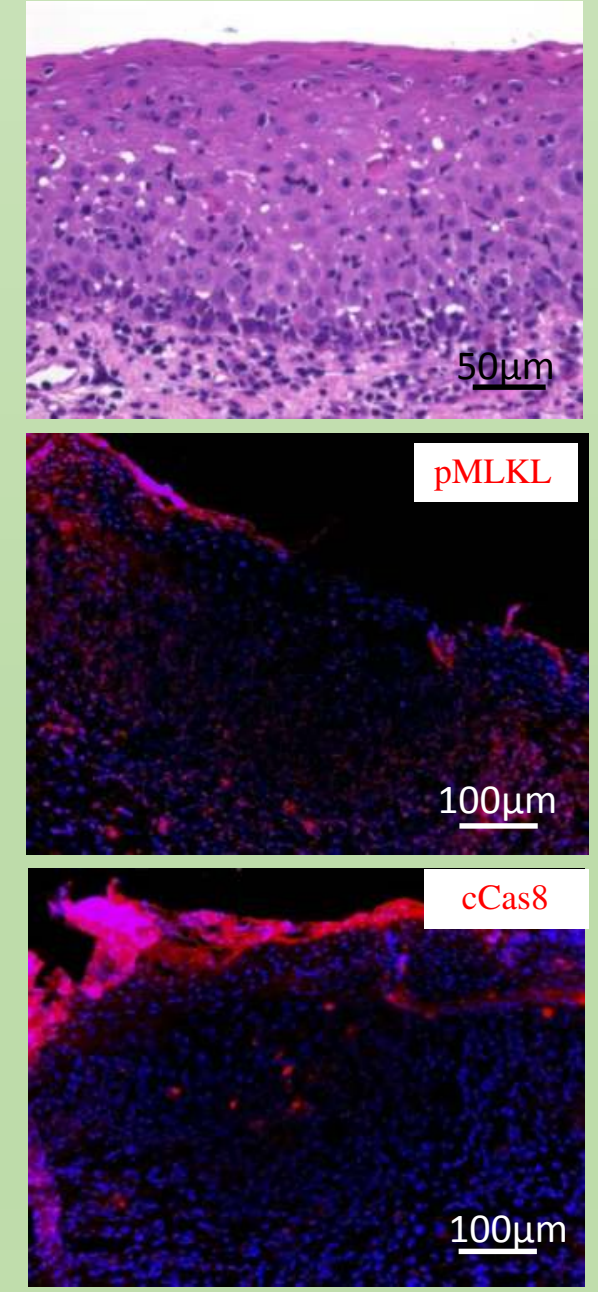

Frequency of cell death in epidermis

\begin{tabular}{cccc} 
& HE $(\%)$ & pMLKL $(\%)$ & cCas8 (\%) \\
\hline SJS & $13.43 \pm 5.39$ & $3.78 \pm 2.70$ & $17.80 \pm 4.94$ \\
EM & $1.02 \pm 1.38$ & $1.47 \pm 2.56$ & $1.09 \pm 2.15$
\end{tabular}

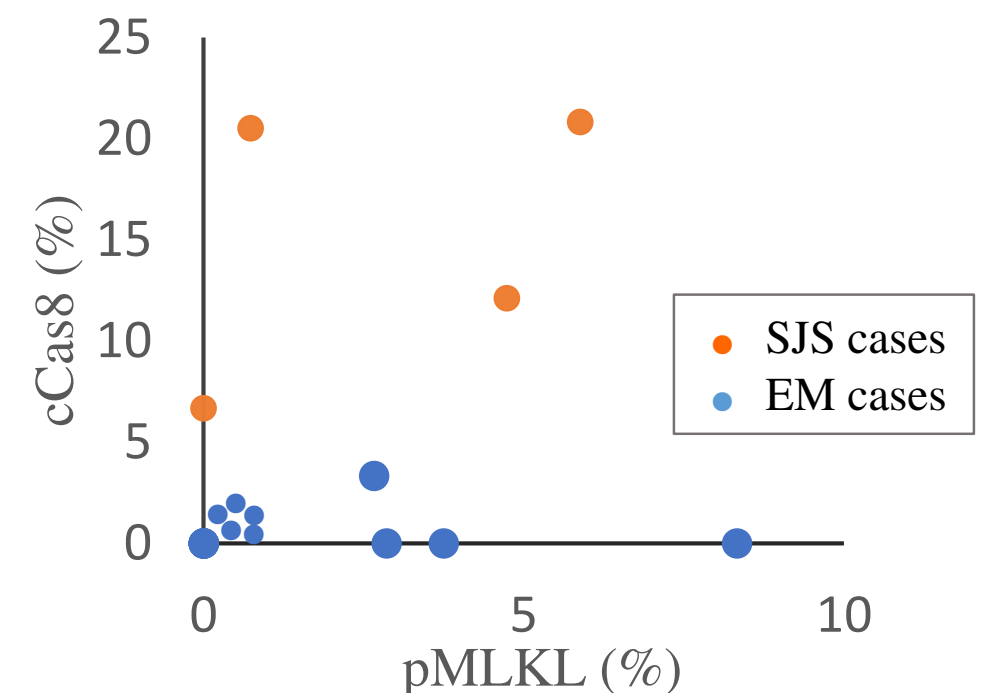

In 4 all cases diagnosed as SJS, both of pMLKL and cCas 8 cell were positive. In contrast, it was only 1 case that both pMLKL and cCas8 were positive and either pMLKL or cCas8 was positive in 4 cases in EM. The number of pMLKL $(+)$ plus cCas $8(+)$ cell were statistically higher than the number of death keratinocyte detected by HE staining.

\section{Conclusion}

Both of necroptosis were apoptosis are occurred in keratinocyte death in SJS. Although, in EM, both of necroptosis and apoptosis were shown, the frequency of cell death was lower than SJS/TEN. It is suggested that the frequency of cell death is important for differentiation between SJS and EM. It is expected that pMLKL and cCas8 are useful for early diagnosis of SJS. 